\title{
Multi-agent technologies in economics
}

\author{
Javier Bajo ${ }^{1}$ Philippe Mathieu $^{2}$ María José Escalona $^{3}$
}

\author{
${ }^{1}$ Departamento de Inteligencia Artificial, \\ Universidad Politécnica de Madrid, Madrid, \\ Spain \\ ${ }^{2}$ Université de Lille, Villeneuve-d'Ascq, France \\ ${ }^{3}$ ETS Ingeniería Informática, Universidad de \\ Sevilla, Seville, Spain \\ Correspondence \\ Javier Bajo, Departamento de Inteligencia \\ Artificial, Universidad Politécnica de Madrid, \\ Madrid, Spain. \\ Email: jbajo@fi.upm.es
}

\begin{abstract}
Summary
This paper summarizes some of the trends in the use of multi-agent technologies in economics. Multiple agent systems, fuzzy sets and neural networks are critical tools used to investigate the emerging economics research agenda related to data mining, dynamic markets stock selection and bank stress testing. This paper reviews the contributions of four such examples.
\end{abstract}

\section{KEYWORDS}

distributed systems, economics, intelligent systems, multi-agent systems
Increasingly, applications in economics, finance and markets are using tools based on artificial intelligence, including multi-agent systems, fuzzy sets, genetic algorithms, neural networks and other approaches, in order to garner a better understanding of the emerging issues. Issues such as the interaction of different markets (e.g. Widiputra, Pears, Serguieva, \& Kasabov, 2009), the automation of explanations (e.g. Daniels \& Caron, 2009) and risk analysis (e.g. Ballini, Mendonça, \& Gomide, 2009; Canedo \& Jaramillo, 2009) can require multiple tools in order to capture the phenomena. Further, it is becoming increasingly important for research to cross disciplinary boundaries in order to access emerging research.

This issue of Intelligent Systems in Accounting, Finance and Management presents a special issue based on papers from the International Conference on Practical Applications on Agents and Multi-Agent Systems (PAAMS 2016) held in Salamanca, Spain, 1-3 June 2016. PAAMS provides an international forum to present and discuss the latest scientific developments and their effective applications, to assess the impact of the approach, and to facilitate technology transfer. PAAMS started as a local initiative, but it has since grown to become the international yearly platform to present, to discuss and to disseminate the latest developments and the most important outcomes related to real-world applications. It provides a unique opportunity to bring multidisciplinary experts, academics and practitioners together to exchange their experience in the development and deployment of agents and multi-agent systems. PAAMS intends to bring together researchers and developers from industry and the academic world to report on the latest scientific and technical advances on the application of multi-agent systems, to discuss and debate the major issues, and to showcase the latest systems using agent-based technology. It will promote a forum for discussion on how agent-based techniques, methods and tools help system designers to accomplish the mapping between available agent technology and application needs. Other stakeholders should be rewarded with a better understanding of the potential and challenges of the agent-oriented approach.

The conference is organized by the Bioinformatics, Intelligent System and Educational Technology Research Group (http://bisite. usal.es/) of the University of Salamanca. During recent years, artificial intelligence systems applied to accounting, financing and management have gained relevance (Albanis \& Batchelor, 2007; Almejalli, Dahal, \& Hossain, 2007; Aragonés, Blanco, \& Estévez, 2007; Ballini et al., 2009; Canedo \& Jaramillo, 2009; Daniels \& Caron, 2009; Di Napoli, Santamaria, \& Rossi, 2017; Galeshchuk, 2017). This special issue is based on selected, expanded and significantly revised versions of the best papers presented at the conference focused on distributed artificial intelligence systems in accounting and financing.

In the first paper, Di Napoli et al. (2017) present a decision support system aimed at helping involved stakeholders to make decisions to plan passengers' transportation in the city and also to evaluate the consequences for the city if the plans are really implemented. Cruise tourism represents a strategic sector for the economic growth of several countries impacting on different direct and indirect markets. The arrival of cruises in a city represents an unmissable opportunity to increment its tourist market penetration. The proposed system is designed according to the multi-agent paradigm, allowing one to easily manage the necessary coordination among different entities and data sources that are usually distributed and need to cooperate to provide useful suggestions. In addition, a prototype of a web-based application is provided to end users, so that it can run on heterogeneous platforms, and it can be easily accessed by different users from different devices, as is the case for the application domain considered. The novelty of the approach proposed in this work is to adopt a system design methodology that allows one to clearly separate the different 
knowledge sources and domain models that contribute to come up with helpful suggestions in the chosen application domain. In fact, the choice to integrate web and multi-agent technologies with a decision support system allows one to include in the system static and dynamic information regarding the city and the port that is crucial in the tourism sector, where decisions usually involve the coordination of different authorities and stakeholders to suggest cruise tourism policies that minimize the negative impact of cruise tourism.

In the second paper, Veryzhenko, Arena, Harb, and Oriol (2017) focus on the French cancel order tax implemented on 1 August 2012. They evaluate the effectiveness of the modified tax with no exemptions and analyse its impact on market quality measured by liquidity, volatility, and efficiency. Additionally, the work presented in the paper raises the question of whether this tax leads to reduction of high-frequency trading (HFT) activities and a declining in trading volume. The authors run two experiments in the agent-based artificial market: (i) different tax regimes and (ii) tax introduced in the middle of a trading period. The paper provides regulators with insights on the HFT tax based on the test results of different market rules not yet implemented. The authors report that introduction of an order cancelling tax reduces HFT activities only slightly, but it significantly affects market liquidity, increases market volatility and deteriorates the market efficiency. Finally, the authors conclude that it is difficult to dissuade investors from entering into unproductive trades and eliminate negative outputs of HFT (such as price manipulations) through tax without altering the benefits of HFT, like liquidity provision and efficient price discovery.

In the paper 'Technological bias in exchange-rate market', Galeshchuk (2017) focuses on demonstrating that traders can leverage technological bias for financial market forecasting. Prediction of exchange-rate markets has been a topic of debate in economic literature since the late 1980s. The paper demonstrates that those traders who adapt faster to the changes in market innovations will get excess returns. To support this hypothesis, the performance of deep learning methods, a shallow neural network with baseline prediction methods, and a random walk model using daily closing rate between three currency pairs is compared: Euro and US dollar (EUR/USD), British pound and US dollar (GBP/USD), and US dollar and Japanese yen (USD/JPY). The results demonstrate that deep learning achieves higher accuracy than alternate methods. The shallow neural network outperforms the random walk model but cannot surpass ARIMA accuracy significantly. The paper discusses possible outcomes of technological shift for financial market development conforming also to adaptive market hypothesis.

In the last paper, Rivas, Parras-Gutiérrez, Merelo, Arenas, and García-Fernández (2017) target the problem of web browsers as agents that can download a web page containing a set of data and the code of an evolutionary algorithm. This way, browsers can execute that algorithm, which has been designed to make evolve artificial neural nets. These neural nets can be applied to many tasks, such as, for instance, forecasting an economic time series. jsEvRBF is a timeseries forecasting method based on a genetic algorithm and neural nets. Written in JavaScript language, it can be executed in most web browsers. Consequently, everybody can participate in the experiments, and scientists can take advantage of the presently available browsers and devices as computation environments. This is also a great challenge, as the language support and performance varies from one browser to another. In their paper, jsEvRBF has been tested in a volunteer computing experiment, and also in a single-browser one. Both experiments are related to forecasting currencies exchange, and the results show the viability of the proposal.

The research presented at PAAMS is consistent with some of the many trends in the use of multi-agent technologies in economics for issues including banking, finance, and economic markets. There, multiple agent technologies are being used, including fuzzy sets and their applications (e.g. Ballini et al., 2009; Leon, Machado, \& Murcia, 2016), neural networks (Aragonés et al., 2007; Resta, 2016), genetic algorithms (Hoogs, Kiehl, Lacomb, \& Senturk, 2007), and evolutionary computing (Lamejalli et al., 2007) that are a critical part of the emerging economics research agenda. This paper also summarizes some of that emerging literature, including the impact on data mining (e.g. Lahmiri, 2016), dynamic markets (Widputra et al., 2009), stock selection classification (e.g. Albanis \& Batchelor, 2007) and stress testing in the banking system (Canedo \& Jaramillo, 2009). As such, it is consistent with research in the emerging areas of computational and multi-agent economics and other cross-disciplinary research.

\section{ACKNOWLEDGEMENTS}

We would like to thank all the contributing authors and the reviewers for their hard and highly valuable work. Their work has helped to contribute to the success of this special issue. Finally, the SURF project associated. We hope the reader will share our joy and find this special issue very useful.

This work has been carried out by the project Intelligent System for Integrated and Sustainable Management of Urban Fleets (SURF), TIN2015-65515-C4-3-R, Ministerio de Economía y Competitividad (Spain), Fondos Feder.

\section{REFERENCES}

Albanis, G., \& Batchelor, R. (2007). Combining heterogeneous classifiers for stock selection. Intelligent Systems in Accounting, Finance and Management, 15, 1-21.

Almejalli, K., Dahal, K., \& Hossain, M. (2007). Intelligent traffic control decision support system. In M. Giacobini (Ed.), Applications of Evolutionary Computing (Vol. 4448Lecture notes in computer science) (pp. 688-701). Berlin: Springer.

Aragonés, J. R., Blanco, C., \& Estévez, P. G. (2007). Neural network volatility forecasts. Intelligent Systems in Accounting, Finance and Management, 15, 107-121.

Ballini, R., Mendonça, A. R. R., \& Gomide, F. (2009). Evolving fuzzy modelling in risk analysis. Intelligent Systems in Accounting, Finance and Management, $16,71-86$.

Canedo, J. M. D., \& Jaramillo, S. M. (2009). A network model of systemic risk: Stress testing the banking system. Intelligent Systems in Accounting, Finance and Management, 16, 87-110.

Daniels, H. A. M., \& Caron, E. A. M. (2009). Automated explanation of financial data. Intelligent Systems in Accounting, Finance and Management, 16, 5-19.

Di Napoli, C., Santamaria, P., \& Rossi, S. (2017). A web-based multi-agent decision support system for a city-oriented management of cruise arrivals. Intelligent Systems in Accounting, Finance and Management, 24. this issue 
Galeshchuk, S. (2017). Technological bias at the exchange rate market Intelligent Systems in Accounting, Finance and Management, 24. this issue

Hoogs, B., Kiehl, T., Lacomb, C., \& Senturk, D. (2007). A genetic algorithm approach to detecting temporal patterns indicative of financial statement fraud. Intelligent Systems in Accounting, Finance and Management, $15,41-56$

Lahmiri, S. (2016). Features selection, data mining and financial risk classification: A comparative study. Intelligent Systems in Accounting, Finance and Management, 23, 265-275.

Leon, C., Machado, C., \& Murcia, A. (2016). Assessing systemic importance with a fuzzy logic inference system. Intelligent Systems in Accounting, Finance and Management, 23, 121-153.

Resta, M. (2016). Enhancing self-organizing map capabilities with graph clustering: An application to financial markets. Intelligent Systems in Accounting, Finance and Management, 23, 21-46.
Rivas, V., Parras-Gutiérrez, E., Merelo, J., Arenas, M., \& García-Fernández, P. (2017). Time series forecasting using evolutionary neural nets implemented in a volunteer computing system. Intelligent Systems in Accounting, Finance and Management, 24. this issue

Veryzhenko, I., Arena, L., Harb, E., \& Oriol, N. (2017). Time to slow down for high-frequency trading? Lessons from artificial markets. Intelligent Systems in Accounting, Finance and Management, 24. this issue

Widiputra, H., Pears, R., Serguieva, A., \& Kasabov, N. (2009). Dynamic interaction networks in modelling and predicting the behaviour of multiple interactive stock markets. Intelligent Systems in Accounting, Finance and Management, 16, 189-205. 